\title{
The Study of AODV Routing Protocol in Vehicular Ad-hoc Sensor Networks
}

\author{
S. Mohamad Suhaili*, N. R. A. Marzuki ${ }^{\dagger}$, M. N. Jambli ${ }^{\dagger}$, H. Lenando ${ }^{\dagger}$, J. Abdullah ${ }^{\dagger}$ \\ * Centre for Pre-University Studies, Universiti Malaysia Sarawak, 94300 Sarawak, Malaysia \\ mssinarwati@preuni.unimas.my \\ ${ }^{\dagger}$ Faculty of Computer Science \& Information Technology, Universiti Malaysia Sarawak, 94300 Sarawak, Malaysia \\ raszamierra@gmail.com,jmnazim@ fit.unimas.my, cool@ fit.unimas.my, ajohari@ fit.unimas.my
}

\begin{abstract}
In VASNETs, each vehicle carries an intelligent node, which sense the real phenomena and collects the required data. There are many routing protocols that have been proposed and assessed the efficiency of VANETs. However, there are only several research about performance evaluation of routing protocol in VASNETs. In order to fill that gap, in this research we have undergo the performance evaluation of AODV routing protocol in VASNETs. The experiment was setup in order to investigate the effect of different simulation area towards increasing velocity and number of mobile nodes. The tools that been used for this purpose is AVRORA simulation tool. Based on the simulation results obtained, the performance of AODV is analyzed and compared in different sizes of simulation area. The simulation results show the significant different in the variation of simulation area in term of the percentage of packet loss in VASNET.
\end{abstract}

Index Terms-Vehicular Ad-hoc Sensor Networks, Ad-hoc OnDemand Distance Vector, Performance Study, Simulation.

\section{INTRODUCTION}

Recently, extensive development of sensor application has been conducted on Vehicular Ad-hoc Sensor Networks (VASNETs) due to the wide potential usage of sensors that focusses on the transportation system. VASNETs play an important role in future automotive development since it has the capability of providing the wide range of services from safety, comfort, telematics and infotainment [1]. In VASNETs, normally a large number of sensor nodes is deployed to utilize sensor nodes on vehicles such as in Vehicular Ad-hoc Networks (VANETs) whic are rapidly emerging research field and considered essential for cooperative driving among vehicles on the road [2]. Such networks can be used to improve road safety in which vehicles are equipped with some short-range and medium-range wireless communication. VASNETs use the same principle with VANETs but inherits its characteristics from both Wireless Sensor Networks (WSNs) and VANETs.

In VASNET, there are different state of sensors including a Rode Side Sensor (RSS) in static node, a mobile node sensor as a Vehicular Node (VN) and Base Station (BS) sensor [3]. The sensors installed on vehicle as VN may have limited communication range with the ground sensors such as RSS and BS sensors as shown in Fig. 1 where the sensors on vehicle can be integrated with the roadside unit and cellular base station to improve the communication to support road safety and traffic management. Therefore, the different size of areas is needed for extending the signal transmission coverage

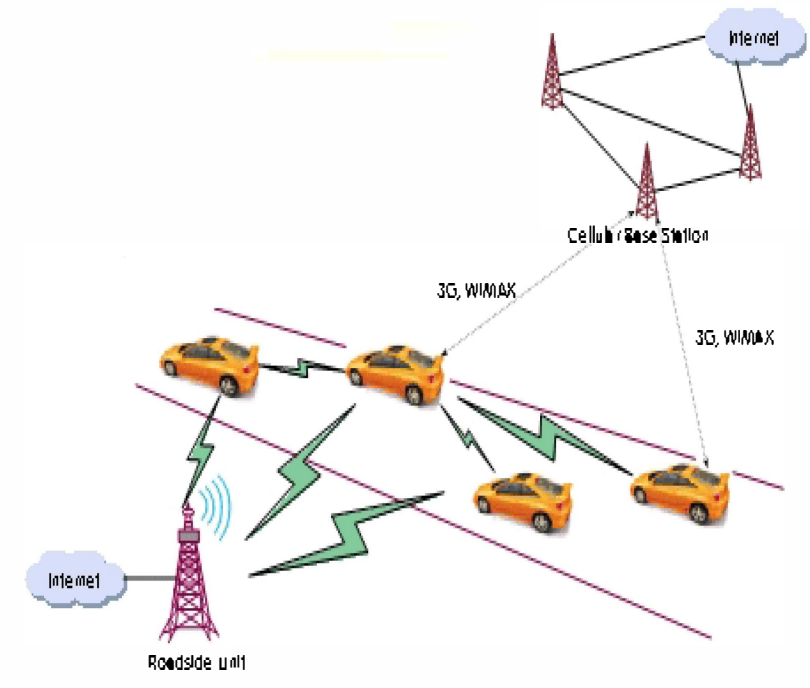

Fig. 1. The architecture of VASNETs in supporting traffic management

and helps to support communication between VN, RSS and BS sensors. Although each sensor node has the ability to increase its transmission power, but still it has several constraints due to the limited processing power, restricted bandwidth, memory and energy of sensor node [4]. Therefore, it is essential to study the performance of routing protocol for VASNETs in respect to different sizes of deployment areas.

In this paper, the performance of Ad-hoc On-Demand Distance Vector (AODV) routing protocol [5] is evaluated on different sizes of simulation area in VASNETs. We analyze the impact of different number and speed of mobile nodes on the average percentage of packet loss when different sizes of simulation areas are used as the deployment area of VASNETs. This research work is based on the simulation where the Avrora network simulator is used to simulate different mobility settings for VASNETs. The rest of the paper is organized as follows. In Section II, we explain the recent related work on the performance evaluation of AODV. Then, the AODV routing protocol description is summarized in section III. Next, the simulation environment and performance metrics are described in Section IV. We present the experimental results and the discussion on the findings in Section V. Finally, Section VI concludes the paper and outlines the future work. 